NASA Technical Memorandum 87329

AIAA-86-1079

\title{
An Efficient Method for Solving the Steady Euler Equations
}

Meng-Sing Liou

Lewis Research Center

Cleveland, Ohio

(NASA-TM-87329) AN EEFICIENT METHOD FOR

N87-11543

SOLVING THE STEADY EULER EQUAIICNS (NASA)

$18 \mathrm{p}$

CSCL $12 \mathrm{~A}$

$63 / 64 \quad \begin{array}{ll}\text { Unclas } \\ 43967\end{array}$

Prepared for the

4th Joint Fluid Mechanics, Plasma Dynamics and Lasers Conference cosponsored by the American Institute of Aeronautics and Astronautics and the American Society of Mechanical Engineers

Atlanta, Georgia, May 12-14, 1986 
AN EFFICIENT METHOD FOR SOLVING THE STEADY EULER EQUATIONS*

\author{
Meng-Sing Liou ${ }^{\dagger}$ \\ National Aeronautics and Space Administration \\ Lewis Research Center \\ Cleveland, Ohio 44135
}

\section{Abstract}

The present paper shows an efficient numerical procedure for solving a set of nonlinear partial differential equations, specifically the steady Euler equations. Solutions of the equations were obtained by Newton's linearization procedure, commonly used to solve the roots of nonlinear algebraic equations. In application of the same procedure for solving a set of differential equations we give a theorem showing that a quadratic convergence rate can be achieved. While the domain of quadratic convergence depends on the problems studled and is unknown a priori, we show that first- and second-order derivatives of flux vectors determine whether the condition for quadratic convergence is satisfled. The first derivatives enter as an implicit operator for yielding new iterates and the second derivatives indicates smoothness of the flows considered. Consequently flows involving shocks are expected to require larger number of iterations.

First-order upwind discretization in conjunction with the Steger-Warming flux-vector splitting is employed on the implicit operator and a diagonal dominant matrix is resulted. However the explicit operator is represented by first- and second-order upwind differencings, using both Steger-Warming's and van Leer's splittings. We discuss treatment of boundary conditions and solution procedures for solving the resulting block matrix system. With a set of test problems for one- and two-dimensional flows, we show detailed study as to the efficlency, accuracy, and convergence of the present method.

\section{Introduction}

During the past decades, much progress has been made in the development of efftcient numerical methods for solving unsteady fluid dynamics equations to yleld time-asymptotic steady solutions. The most noted algorithms have been the explicit schemes such as the predictor-corrector scheme by MacCormack ${ }^{1}$ and more recently the application of Runge-Kutta method by Jameson et a1.,2 and the implicit ADI schemes such as those due to Briley and McDonald 3 and Beam and Warming. 4 Various strategies have been utilized to accelerate computational efficiencies, e.g., multigrid, 5 vectortzation, etc.

The present paper proposes a different approach wherein the steady-state solutions are obtained by solving the steady equations, rather than through the time marching approach as

*This work was initiated while the author was a Visiting Associate Professor at The University of Michigan, Dept. of Aerospace Engineering. Ann Arbor, Michigan 48109 funded by AFOSR Grant AFOSR-84-0327.

tMember AIAA.

This paper is declared a work of the U.S. Government and therefore is not subject to domestic copyright. mentioned above. We are motivated in this research by asking ourselves the following questions:

(1) Is time marching technique more efficient?

(2) Can a mathematical basis (even approximated) be established to indicate fast convergence of a steady-equations solver?

(3) How can it be done and what are the differences between the unsteady-and steady-equations approaches?

Obviously the first question is general and the answer is dependent upon circumstances considered. In fact is is well known that a spacemarching approach is much more efficient than the time-marching approach for solving steady, fully supersonic problems. Therefore it is important to investigate the detalled differences in concept and mathematics between the two approaches. We note that this research was motivated by the paper by MacCormack ${ }^{6}$ in which the Idea of using Newton's method was mentioned while again unsteady equations were used in applications. In this paper we attempt to shed some light on the questions (2) and (3) together with some applications to various problems.

We apply the Newton method, which is commonly used for iteratively solving roots of nonlinear algebraic equations, to find solutions to the steady Euler equations. Consequently quadratic* convergence can be achieved provided the iterative sequence is sufficiently close to the true solutions. Upwind differencing is used to approximate the derivatives and a diagonal dominant matrix system can be obtained, thereby stabilizing the t teration process. Several investigations $b-9$ utilizing Newton's iteration between two time levels and upwind-differencing, have shown great success in efficientiy arriving time-asymptotic steady solutions. It is noted that the present Newton linearization form is recovered if we let $\Delta t=\infty$ in the implicit unsteady procedures, e.g. , Beam-Warming's unfactored form. 4 While some analytical similarities may exist between the steady and unsteady approaches, we believe that there are differences in detail which are manifested in the convergence history. The iteration in general consists of two stages, the initial stage beginning with an arbitrary guess sets out to home in the domain of Newton's quadratic convergence and the second stage locks in this range with rapid convergence.

We show in section 1 a theorem indicating quadratic convergence for the case of differential equations. A condition for the doma in of quadratic convergence, $\Omega_{2}$, indicates that whether an

*Quadratic convergence means the egror $\|$ ell is reduced quadratically, i.e., $\|e\|_{n+1} /\|e\|_{n}^{2}=C$. 
approximation lies in $\Omega_{2}$ depends on rate of change and smoothness of flow vectors, hence is problem dependent. The choice of spatial differencing is of particular importance for the present method and is given in section 2 . Section 3 gives treatment of boundary conditions. The aigebraic system of equations resulting from sections 2 and 3 is then summarized; the solution strategies are discussed in section 4 . Finally we show in section 5 the convergence of calculated solutions by comparing with exact solutions in one- and twodimensional problems. The flux splittings by Steger and Warming 11 and van Leer 12 are employed. Pertinent issues such as accuracy and convergence rate are discussed.

\section{Newton's Method}

We begin by considering the steady Euler equations in conservation-law form and Cartesian coordinates:

$$
\Psi(U)=D_{x} F+D_{y} G+D_{z} H=0
$$

where $\Psi=\left\{\Psi_{1}, \Psi_{2}, \ldots \ldots, \Psi_{5}\right\}$ represents five (5) partial differential equations, each is a function of the conservative variables $U=\{\rho(1, u, v, w, E)\}$. Here $\rho$ is the density, $(u, v, w)$ are Cartesian components of the velocity, and $E$ is the sum of specific internal and kinetic energy. The flux vectors are shown below for completeness.

$F=\left(\begin{array}{c}\rho u \\ \rho u^{2}+p \\ \rho u v \\ \rho u w \\ (\rho E+p) u\end{array}\right), G=\left(\begin{array}{c}\rho v \\ \rho v u \\ \rho v^{2}+p \\ \rho v w \\ (\rho E+p) v\end{array}\right), H=\left(\begin{array}{c}\rho w \\ \rho w u \\ \rho w v \\ \rho w^{2}+p \\ (\rho E+p) w\end{array}\right)$

In Eq. (1a) we choose the notation

$$
D_{x} \equiv \frac{\partial}{\partial x}, \quad D_{y} \equiv \frac{\partial}{\partial y}, \quad D_{z} \equiv \frac{\partial}{\partial z} .
$$

Also perfect gas is assumed so that flux vectors are homogeneous function of $U$ of degree one.

$\Psi(U)=0,1 . \mathrm{e}$.,

Assuming $U^{\star}$ is a root (solution) of

$$
\Psi\left(U^{*}\right)=0
$$

Since the system of equations in Eq. (1a) is noninear and coupled, thus we are obliged to use approximate method to iteratively arrive at approximate solution to $U^{\star}$. The Newton iterative method having quadratic convergence for solving algebraic equations also can be extended to solve differential equations. The Newton procedure starts by keeping the first-order term in Taylor's series expansion of $\Psi\left(U^{n+1}\right)$ and forces $U^{n+1}$ to satisfy Eq. (1a). We have

$$
D_{u} \Psi^{n} \cdot \delta U=-\Psi\left(U^{n}\right), \quad n=0,1,2, \ldots .
$$

where $\delta U=U^{n+1}-U^{n}$. The differential operator $D_{U} \Psi$ working on sU is given by

$$
D_{u} \Psi^{n} \equiv \frac{\partial \Psi}{\partial U}\left(U^{n}\right)=\left(D_{x} A+D_{y} B+D_{z} C\right)^{n}
$$

here the Jacobian matrices are $A=D_{U} F, B=D_{U} G$, and $\mathrm{C}=\mathrm{D}_{\mathrm{u}} \mathrm{H} .4$

Equation (3) is the basic equation for deriving the new terate $u^{n+1}$ and is, in fact, Identical to that derived in Ref. 4 when $\Delta t=\infty$ is taken there.

For the quasi one-dimensional Euler equations,

$$
D_{x} F+S=0
$$

where

$$
S(U) \equiv\left[F-\left(\begin{array}{l}
0 \\
P \\
0
\end{array}\right)\right] \cdot K, \quad K(X) \equiv D_{x} \text { (area)/area }
$$

The Newton procedure yields

$$
D_{u} \Psi^{n} \delta U^{n}=D_{x}\left(A^{n} \cdot \delta U^{n}\right)+B^{n} \cdot \delta U^{n}=-\left(D_{x} F+S\right)^{n}
$$

Here we define $B$ as

$$
B \equiv \frac{\partial S}{\partial U}=K\left(\begin{array}{ccc}
0 & 1 & 0 \\
-u^{2} & 2 u & 0 \\
u\left[(\gamma-1) u^{2}-\gamma E\right] & \gamma E-3(\gamma-1) u^{2} / 2 & \gamma U
\end{array}\right)
$$

We turn now to study the convergence property of the Newton method. Assuming that $\Psi$ is twice differentiable and that the root (solution) $U^{*}$ that we are seeking is a simple root (multiplicity $=1)$. Then we have $D_{u} \Psi\left(U^{*}\right) \neq 0$ and $D_{u} \Psi(U) \neq 0$ for all $U$ in a certain neighborhood of $U^{*}$ (see Ref. 10). have

Using (,) to denote partial derivatives, we

$$
\begin{aligned}
& \Psi_{1, j} \equiv \frac{\partial \Psi_{1}}{\partial U_{j}} \\
& \Psi_{i, j k} \equiv \frac{\partial^{2} \Psi_{1}}{\partial U_{j} \partial U_{k}} \quad 1, j, k=1,2, \ldots ., D
\end{aligned}
$$

Now expand $\Psi\left(U^{*}\right)$ about $\Psi\left(U^{n}\right)$ in Taylor's series

$$
\begin{aligned}
& \Psi_{i}\left(u^{*}\right)=\Psi_{i}\left(u^{n}\right)+\sum_{j} \Psi_{1, j}\left(u^{n}\right)\left(u_{j}^{*}-u_{j}^{n}\right) \\
& +\frac{1}{2} \sum_{j} \sum_{k} \Psi_{i, j k}(v)\left(u_{j}^{*}-u_{j}^{n}\right) \cdot\left(u_{k}^{*}-u_{k}^{n}\right)
\end{aligned}
$$


for $1=1,2, \ldots, P$, where $v$ lies in the interval containing $U^{\star}$ and $u^{n}$. Since

$$
\begin{aligned}
\sum_{j} \Psi_{i, j}^{n}\left(u_{j}^{*}-u_{j}^{n+1}\right)= & \sum_{j} \Psi_{i, j}^{n}\left(u_{j}^{*}-u_{j}^{n}\right) \\
& -\sum_{j} \Psi_{1, j}^{n}\left(u_{j}^{n+1}-u_{j}^{n}\right)
\end{aligned}
$$

We have, after applying Eqs. (3) and (7),

$$
\begin{aligned}
& \sum_{j} \Psi_{i, j}^{n}\left(u_{j}^{*}-u_{j}^{n+1}\right) \\
& \quad=-\frac{1}{2} \sum_{j} \sum_{k} \Psi_{i, j k}(v)\left(u_{j}^{n}-u_{j}^{*}\right)\left(u_{k}^{n}-u_{k}^{*}\right)
\end{aligned}
$$

Let $c^{n}$ be the error at the nth iteration,

$$
\varepsilon^{n} \equiv u^{n}-u^{*}
$$

and denote $A=\left\{a_{1 j}\right\}, B=\left\{b_{1 j}\right\}, C=\left\{c_{1 j}\right\}$. Substituting Eq. (4) in Eq. (8) yleids

$\sum_{j}\left(D_{x} a_{i j}^{n}+D_{y} b_{i j}^{n}+D_{z} c_{i j}^{n}\right) \varepsilon_{j}^{n+1}=-\frac{1}{2} \sum_{j} \sum_{k} \Psi_{i, j k}(v) \varepsilon_{j}^{n} \varepsilon_{k}^{n}$

Integrating over an arbitrary volume $\Delta a=\Delta x \Delta y \Delta z$ (here $\Delta x, \Delta y, \Delta z$ are not taken to be grid spacings) and making use of the Gauss theorem, we find

$$
\begin{aligned}
& \sum_{j} \int \varepsilon_{j}^{n+1}\left(a_{i j} d y d z+b_{i j} d x d z+c_{1 j} d x d y\right) \\
& =-\frac{1}{2} \sum_{k} \sum_{j} \int c_{j}^{n} \varepsilon_{k}^{n}\left(a_{1 j, k} d y d z+b_{i j, k} d x d z\right. \\
& \left.+c_{i j, k} d x d y\right)(v)
\end{aligned}
$$

where $(, k)$ as before denotes differentiation with respect to $U_{k}$.

By the mean value theorem the equation becomes

$$
\begin{aligned}
\sum_{j}\left(\frac{a_{1 j}(\xi)}{\Delta x} \varepsilon_{j}^{n+1}(\xi)\right. & \left.+\frac{b_{i j}(n)}{\Delta y} \varepsilon_{j}^{n+1}(n)+\frac{c_{1 j}(\zeta)}{\Delta z} \varepsilon_{j}^{n+1}(\zeta)\right) \\
= & -\frac{1}{2} \sum_{k} \sum_{j}\left(\frac{1}{\Delta x} a_{i j, k^{\prime}}\left(\xi^{\prime}\right) \varepsilon_{j}^{n}\left(\xi^{\prime}\right) \varepsilon_{k}^{n}\left(\xi^{\prime}\right)\right. \\
& +\frac{1}{\Delta y} b_{i j, k^{\prime}}\left(n^{\prime}\right) \varepsilon_{j}^{n}\left(n^{\prime}\right) \varepsilon_{k}^{n}\left(n^{\prime}\right) \\
& \left.+\frac{1}{\Delta z} c_{i j, k^{\prime}}\left(\zeta^{\prime}\right) \varepsilon_{j}^{n}\left(\zeta^{\prime}\right) \varepsilon_{k}^{n}\left(\zeta^{\prime}\right)\right)
\end{aligned}
$$

where $\xi, n, \zeta, \xi^{\prime}, \eta^{\prime}, \zeta^{\prime} \in \Delta a$. We understood that $a_{i j}, b_{i j}$, and $c_{i j}$ on LHS were evaluated at $u^{n}$ and $a_{i j, k}, b_{i j, k}$, and $c_{i j, k}$ on RHS at $V$. Assuming $\Delta \sigma$ smalf' and

$$
\varepsilon_{j}(\xi) \approx \varepsilon_{j}(n) \approx \varepsilon_{j}(\zeta) \text { etc. }
$$

We have

$$
\sum_{j} q_{i j \varepsilon_{j}^{n+1}}=\frac{1}{2} \sum_{k} \sum_{j} q_{i j, k} \varepsilon_{j}^{n} \varepsilon_{k}^{n}
$$

where

$$
\begin{gathered}
q_{i j} \equiv \frac{a_{i j}}{\Delta x}+\frac{b_{i j}}{\Delta y}+\frac{c_{i j}}{\Delta z} \\
q_{1 j, k} \equiv \frac{\partial q_{i j}}{\partial u_{k}}
\end{gathered}
$$

Now for maximum vector norms and corresponding matrix norms we obtain

$$
\left\|e^{n+1}\right\|_{\infty} \leqslant m\left\|_{\varepsilon}^{n}\right\|_{\infty}^{2}
$$

where $m$ is chosen such that

$$
\frac{p}{2}\left\|\frac{\partial Q}{\partial U_{k}}\right\|_{\infty}\left\|_{Q^{-1}}\right\|_{\infty} \leqslant m, \quad \text { for a } 11 \quad 1 \leqslant k \leqslant p
$$

where the square matrix $Q \equiv\left\{q_{j}\right\}$ is assumed invertible and $p$ is the number of equations in $C$. For Euler equations, Eq. (13a) gives, for all k

$\frac{\mathrm{p}}{2}\left\|\left(\frac{\mathrm{A}}{\Delta \mathrm{x}}+\frac{\mathrm{B}}{\Delta y}+\frac{\mathrm{C}}{\Delta \mathrm{z}}\right)^{-1}\right\|_{\infty}\left\|\frac{1}{\Delta x} \frac{\partial A}{\partial U_{k}}+\frac{1}{\Delta y} \frac{\partial B}{\partial U_{k}}+\frac{1}{\Delta z} \frac{\partial C}{\partial U_{k}}\right\|_{\infty} \leqslant m$

A simflar condition for the one-dimensional equations is readily obtained.

$$
\frac{3}{2}\left\|\left(\frac{A}{\Delta x}+B\right)^{-1}\right\|_{\infty}\left\|\frac{1}{\Delta x} \frac{\partial A}{\partial U_{k}}+\frac{\partial B}{\partial U_{k}}\right\|_{\infty} \leqslant m
$$

for $k=1,2,3$.

THEOREM. Suppose that $\Psi(U)=0$ has a root $U^{*}$ and that in the sphere $\Omega_{2}=\left\{U: m\left\|U-U^{\star}\right\|_{\infty} \leqslant 1\right\}$. Assuming $\Psi$ has first and second derivatives such that Eq. (12) is satisfied. Then for all $U^{0} \in \Omega_{2}$, we have
(a) $U^{n} \in \Omega_{2}, \quad n=1,2, \ldots$.
(b) $\lim u^{n}=u^{*}$, $n \rightarrow \infty$
(c) the convergence is quadratic
(d) $U^{\star}$ is unique in $\Omega_{2}$

Proof. Since $\left\|m_{\varepsilon} 0\right\|_{\infty} \leqslant 1$, then by Eq. (12)

$$
\|\dot{m \varepsilon}\|_{\infty}^{1} \leqslant\left\|m_{\varepsilon}\right\|_{\infty}^{2} \leqslant 1
$$


Hence $U^{1} \in \Omega_{2}$. By induction it follows $U^{n} \in \Omega_{2}$, for $\mathrm{n}=1,2,3, \ldots$

Next, repeated use of Eq. (12) gives

$$
\|\varepsilon\|_{\infty} \leqslant m^{-1}\left\|_{m \varepsilon}^{0}\right\|_{\infty}^{2^{n}}
$$

Then result (b) follows immedlately as $n \rightarrow \infty$. Since $u^{0}, u^{1}, u^{2}, \ldots$ is a sequence which converges to ' $U^{\star}$, i.e., in $U^{*} U^{*}, V \rightarrow U^{*}$ as $n \rightarrow \infty$, there exists a constant $C\left(U^{\star}\right) \neq 0$ such that Eq. (12) yields

$$
\left\|\varepsilon^{n+1}\right\|_{\infty}=c\left\|\varepsilon_{\varepsilon}^{n}\right\|_{\infty}^{2}, \quad n \rightarrow \infty
$$

The sequence is, by definition, said to be quadratically convergent. Next we prove the uniqueness for a solution in $\Omega_{2}$.

Suppose there exists another root $V^{\star} \neq U^{\star}$ in $\Omega_{2}$, then $\Psi\left(v^{*}\right)=0$. for $1=1,2, \ldots \ldots$, we write

$$
\begin{aligned}
u_{1}^{*}-v_{i}^{*}=u_{i}^{*}+\Psi_{j}\left(u^{*}\right) & -\left(v_{i}^{*}+\Psi_{i}\left(v^{*}\right)\right) \\
& =u_{i}^{*}-v_{i}^{*}+\sum_{j} \Psi_{i, j}(v)\left(u_{j}^{*}-v_{j}^{*}\right)
\end{aligned}
$$

for $V$ in an intervai containing $\left(U^{*}, V^{*}\right)$. Hence

$$
\left|u_{1}^{*}-v_{1}^{*}\right| \leqslant\left|u_{i}^{*}-v_{1}^{*}\right|+\left\|u^{*}-v^{*}\right\|_{\infty} \sum_{j}\left|\Psi_{1, j}(v)\right|
$$

Since this inequality is true for all $i$,

$$
\left\|u^{*}-v^{*}\right\|_{\infty} \leqslant\left\|u^{*}-v^{\star}\right\|_{\infty}+\left\|u^{*}-v^{*}\right\|_{\infty}\left\|\Psi_{i, j}\right\|_{\infty}
$$

a contradiction, hence (d) is proved.

From Eq. (14), one can readily estimate the number of iterations required for an initial error to be reduced by $10^{-5}$.

$$
n \approx \frac{1}{\log 2} \log \left[\frac{s}{\log \left(1 / m\left\|_{\varepsilon}^{0}\right\|_{\infty}\right)}\right]
$$

We shall note that the domain $\Omega_{2}$ is the estimates for the required closeness of approximation to the true solution in which Newton's quadratic convergence holds. It is function of the gradient of the flux vectors with respect to $U$, i.e., Jacobians, and smoothness of fluxes in the problems concerned.

In genera $1 U^{*}$ is of course unknown, the above conditions, $13(\mathrm{~b})$ or $13(\mathrm{c})$, must be problem dependent and is difficult to verify beforehand. We shall demonstrate through test cases that in reality one need not start with close approximation. A relaxation procedure is used to move approximation progressively toward the doma in $\Omega_{2}$; thereafter a distinctly faster convergence is followed.
After solving a block matrix system (given in section 4), we update the approximation by the equation

$$
u^{n+1}=u^{n}+\omega \delta u^{n}
$$

where the relaxation factor $\omega$ in general can be a constant matrix varying with iteration. The precise variation of $\omega$ for which the iteration will remain stable is not known. A series of numerical experiments for varlous circumstances have been performed and their resuits will be presented later.

We show now the difference between the time-marching and the present approaches. Let $\delta U^{n}=\omega \delta U^{n}$. Since $\omega$ is constant, combination of Eqs. (3) and (15) gives

$$
\begin{aligned}
& D_{u} \Psi^{n} \cdot \delta \hat{U}^{n}=-\omega \Psi^{n} \\
& u^{n+1}=U^{n}+\delta \hat{U}^{n}
\end{aligned}
$$

Hence we see that the present iteration process bears no direct relation to the time-marching approach since a different implicit operator having no time derivative term is involved. However, like the case of time-dependent approach, the solution satisfies $\Psi(U)=0$ as $\delta U \rightarrow 0$.

\section{Spatial Differencing}

Since there can be independent choices of types of differencing for approximating the implicit operator on LHS and explicit operator on RHS of the Eqs. (3) or (6) insofar as stability is maintained, we shall describe them separately. The effects of mixed use of differencing on the convergence rate and accuracy w111 be discussed in section 5 . It will become clear in what follows that it is imperative to use upwind differencing on the implicit operator.

Now we split the Jacobian matrix $A$ as

$$
A=A^{+}+A^{-}
$$

where $A^{+}$and $A^{-}$are matrices derived from the corresponding " + " and "-" split fluxes, $F+$ and $\mathrm{F}^{-}$given by Steger and Warming, 11 specifically

$$
A^{ \pm} \equiv \frac{\partial F^{ \pm}}{\partial U}
$$

These are the true Jacobians of relevant split fluxes and not identical to the matrices resulting from eigenvalue splitting, as given by:

$$
\tilde{A}^{ \pm}=Q^{ \pm} Q^{-1}
$$

where $Q$ is a similarity matrix diagonalizing A. ${ }^{4}$ The complete expression for $A^{ \pm}$and $B^{ \pm}$in twodimenstonal case is given in Appendix $A$.

Now we can write (omitting iteration index $n$ hereafter) 


$$
D_{x}(A \cdot \delta U)=\frac{1}{\Delta x}\left[\Delta^{-} A^{+} \delta U+\Delta^{+} A^{-} \delta U\right]+0(\Delta x)
$$

Here we denote $\Delta^{-}$and $\Delta^{+}$as backward and forward difference operators. Let

$$
|A|=A^{+}-A^{-}
$$

Equation (18) becomes, for the $j$ th grid point,

$$
D_{x}(A \delta U)=\frac{1}{\Delta x}\left\{-A_{j-1}^{+},\left|A_{j}\right|, A_{j+1}^{-}\right\}\left\{\begin{array}{c}
\delta U_{j-1} \\
\delta U_{j} \\
\delta U_{j+1}
\end{array}\right\}
$$

for $j=1,2, \ldots . . J$. Substituting of Eq. (19) in Eq. (6) gives, for one-dimensional equations,

$$
D_{u} \Psi \delta U=T \delta U
$$

where $T$ is a block tridlagonal system $\{T\}$, $\left.\mathrm{T}_{2}, \ldots ., \mathrm{T}_{j}\right\}$ and $\mathrm{T}_{j}=1 / \Delta x\left\{-\mathrm{A}_{j}-1,\left|A_{j}\right|\right.$ $\left.+\Delta x B_{j}, A_{j+1}\right\}$. Also hereafter $\delta U$ denotes $\left\{\delta U_{0}\right.$, $\left.\delta U_{1}, \ldots . . \delta U_{j} . ., \delta U_{j}, \delta U_{j}+1\right\} ; \delta U_{0}$ and $\delta U_{J+1}$ are evaluated at boundaries.

Following the same procedures in deriving Eqs. (12) and (13), we now write their counterparts for the discretized algebraic system,

$$
\left\|T^{-1}\right\|_{\infty} \| \text { discretized } \Psi_{1, j k} \|_{\infty} \leqslant m
$$

It is obvious that whether the condition for quadratic convergence is satisfied and fast convergence can be realized depends on (1) the property of the matrix $T$ resulting from discretization of the implicit operator, and (2) the smoothness of flux vector as given in $\Psi_{i, j k}$. Therefore a wellconditioned implicit operator is required. Since the first-order upwinding yields a matrix which is diagonally dominant and hence well-conditioned. It also has minimum bandwith for coupling neighboring points, hence making boundary conditions felt in each iteration step. Therefore we shall use only first-order upwinding for the implicit operator while having the liberty of utilizing different differencings for the explicit operator.

We note that despite the central differencing possesses properties of being simple and higherorder accurate with the same bandwith, it however leads to vanishing of diagonal terms and hence losing diagonal dominance. Consequently the iterative sequence will likely diverge.

We turn now to the differencing of the explicit operator. First-order and second-order upwinding bascd on flux splitting are employed on uniform grids, we have

$$
D_{x} F=D_{x}\left(F^{+}+F^{-}\right)
$$

and

$$
D_{X} F^{ \pm}=\frac{1}{\Delta x}\left(\Delta^{\mp} F^{ \pm}+\frac{\theta}{2} \Delta^{-} \Delta^{+} E^{\mp} F^{ \pm}\right)+0\left[(\Delta x)^{1-\theta}, \Delta x^{2}\right]
$$

where $E$ is the displacement operator. $\theta=0$ and 1 denote first-and second-order accuracy. Similar differencing can be made for $D_{y} G$ and $D_{z} H$ for multidimensional flows. The flux splittings proposed by Steger and Warming 11 and Van Leer 12 are employed in our study. They are given here for completeness (for two-dimenstonal case).

Steger-Warming:

$$
\begin{aligned}
& \text { Let } F_{1}^{+}=2(\gamma-1) \lambda_{1}^{+}+\lambda_{3}^{+}+\lambda_{4}^{+} \\
& F^{+}=\frac{e}{2 \gamma}\left[\begin{array}{l}
F_{1}^{+} \\
u F_{1}^{+}+c\left(\lambda_{3}^{+}-\lambda_{4}^{+}\right) \\
v F_{1}^{+} \\
q^{2} F_{1}^{+}+c u\left(\lambda_{3}^{+}-\lambda_{4}^{+}\right)+c^{2}\left(\lambda_{3}^{+}+\lambda_{4}^{+}\right) / 2(r-1)
\end{array}\right]
\end{aligned}
$$

where $\lambda_{1}^{+}=\left(\lambda_{1}+\left|\lambda_{1}\right|\right) / 2,1=1,2,3,4$ and $\lambda_{1}$ $=\lambda_{2}=u, \lambda_{3}=u+c$, and $\lambda_{4}=u-c ; c=$ speed $b_{f}$ souhd.

$$
\text { Now if } G_{1}^{+}=2(\gamma-1) \lambda_{1}^{+}+\lambda_{3}^{+}+\lambda_{4}^{+} \text {and } v \text { is }
$$
substituted for $u$ in $\lambda_{1}$ above, we have

$$
G^{+}=\frac{\rho}{2 \gamma}\left[\begin{array}{l}
G_{1}^{+} \\
U G_{1}^{+} \\
V G_{1}^{+}+c\left(\lambda_{3}^{+}-\lambda_{4}^{+}\right) \\
\frac{q^{2}}{2} G_{1}^{+}+c v\left(\lambda_{3}^{+}-\lambda_{4}^{+}\right)+c^{2}\left(\lambda_{3}^{+}+\lambda_{4}^{+}\right) / 2(\gamma-1)
\end{array}\right]
$$

Van Leer:

$$
\begin{array}{ll}
F^{+}=F, & M>1 \\
F^{-}=F, & M<-1
\end{array}
$$

and for $|M|<1$

$$
F^{ \pm}=F_{1}^{ \pm}\left[\begin{array}{l}
1 \\
{[(\gamma-1) u \pm 2 c] / \gamma} \\
v \\
{[(\gamma-1) u \pm 2 c]^{2} / 2\left(\gamma^{2}-1\right)+v^{2} / 2}
\end{array}\right]
$$

where

$$
F_{1}^{ \pm}= \pm \rho c\left(M_{x} \pm 1\right)^{2} / 4, \quad M_{x}=u / c
$$


The formula for $G^{ \pm}$are obtained by interchanging $u$ and $v$ and elements 2 and 3 in $F \pm$. Differences of analytical properties of both splitting are given in Ref. 12. We shall discuss their differences in calculated results in section 5 .

Now substituting Eqs. (20) and (22) in

Eq. (6), we have the block tridiagonal system

$$
T \delta U=-f
$$

where $f=\left\{f_{1}, f_{2}, \ldots, f_{j}, \ldots ., f_{j}\right\}$ and

$f_{j}=\frac{1}{\Delta x}\left(\Delta^{-} F_{j}^{+}+\Delta^{+} F_{j}^{-}\right)+\frac{\theta}{2 \Delta x} \Delta^{-} \Delta^{+}\left(F_{j-1}^{+}+F_{j+1}^{-}\right)+S_{j}$

The system needs to be closed by boundary conditions, to be discussed in the next section.

For multidimensional problem, simllar upwinding can be constructed. Assuming equal-spacing grids in two dimensions, $x=j \Delta x, j=0,1,2$, . .,$j+1$ and $y=k \Delta y, k=0,1,2, \ldots$ $k+1$ and $a=\Delta y / \Delta x$. The resuiting block matrix system is written as

$$
M \delta U=-f
$$

where for typewriter ordering, 1.e., on each constant $y$, we have

$$
\begin{gathered}
\delta U=\left\{\delta U_{0}, \delta U_{1}, \ldots ., \delta U_{k}, \ldots ., \delta U_{k+1}\right\} \\
f=\left\{f_{1}, f_{2}, \ldots ., f_{k}, . ., f_{k}\right\}
\end{gathered}
$$

and each element in $\delta U$ and $f$,

$$
\begin{aligned}
& \delta U_{k}=\left\{\delta U_{0 k}, \delta U_{1 k}, \ldots ., \delta U_{j k}, . ., \delta U_{j+1, k}\right\}, \\
& k=0,1,2, \ldots ., k+1 \\
& f=\left\{f_{1 k}, f_{2 k}, \cdot \cdot, f_{j k}, \cdot \ldots f_{j k}\right\}, \\
& k=1,2,3, \ldots \ldots, k
\end{aligned}
$$

The matrix $M$ has the structure

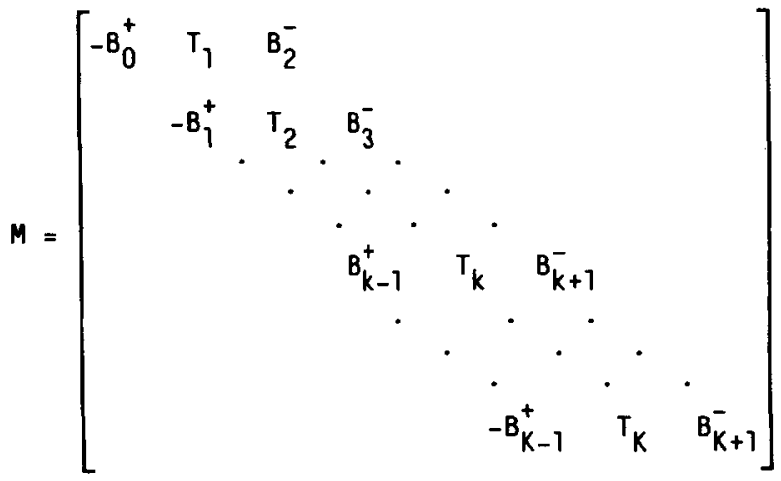

which again is of block tridiagonal form. The element matrices $B \frac{F}{k}$ and $T_{k}$ are found to be of block diagonal and block tridiagonal form respectively, namely
$B_{k}^{ \pm}=\frac{1}{\Delta y}\left[\begin{array}{llll}B_{1 k}^{ \pm} & & & \\ & B_{2 k}^{ \pm} & & \\ & & \cdot & \\ & & \cdot & \\ & & & B_{j k}^{ \pm}\end{array}\right] \quad k=0,1,2, \ldots, k+1$

and

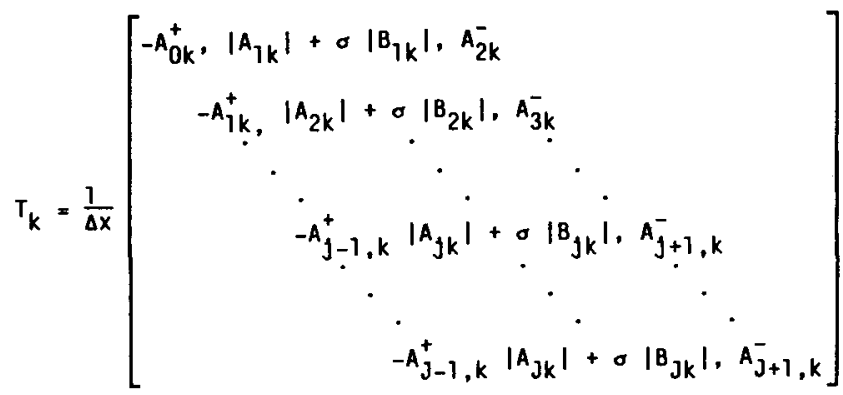

for $k=1,2, \ldots . ., k$. We note that $T_{k}$ is a block tridiagonal system (each constant $y$ ) within a large block tridiagonal system $M$. One can also, by altering the typewriter ordering, construct block tridiagonal system for each constant-x line.

There exists many classical iterative schemes for solving a large system; several strategies are discussed in Ref. 7. We shall outline possible candidates for solving the system. Eq. (26), in section 4 after the boundary conditions are treated. We note here again conditions on the boundary lines $(k=0, k+1, j=0, J+1)$ must be imposed to render the system solvable.

\section{Boundary Condition}

Boundary conditions are treated implicitly and shown below for various cases.

(a) supersonic inflow:

All variables are prescribed and remain unchanged at exterior points,

$$
\begin{gathered}
\delta U=0 \\
\Delta^{-} F^{+}=0
\end{gathered}
$$

(b) supersonic outflow: e.g.

All variables are extrapolated from interior,

$$
\begin{aligned}
& \Delta U=0 \\
& \Delta^{-} F^{+}=0
\end{aligned}
$$

(c) subsonic inflow:

We prescribe $\rho, u$, and $v$ and extrapolate p from neighboring interior points, e.g.. 


$$
\Delta^{+} p=0
$$

(d) subsonic outflow:

The pressure (p) is fixed and the remaining varlables are extrapolated from interior, e.g.,

$$
\begin{aligned}
& \Delta^{-} P=0 \\
& \Delta^{-} u=0 \\
& \Delta^{-} v=0
\end{aligned}
$$

This case is more involved and needs some elaboration. We lllustrate how the boundary terms on LHS are combined with the neighboring interior terms. since $p$ is fixed, we have

$$
\delta \rho E=-\frac{u^{2}}{2} \delta \rho+u \delta(\rho u) \quad(\text { for } 1-D \text { problem) }
$$

Applying the extrapolation (Eq. (32)), we find

$$
\begin{aligned}
A_{J+1}^{-} \delta u_{J+1} & =\left(\begin{array}{ccc}
a_{11} & a_{12} & a_{13} \\
a_{21} & a_{22} & a_{23} \\
a_{31} & a_{32} & a_{33}
\end{array}\right)_{J+1}\left(\begin{array}{c}
-\frac{u^{2}}{2} \delta_{\rho}+u \delta \rho u \\
\delta \rho u \\
\delta \rho
\end{array}\right)_{J+1} \\
& =\left(\begin{array}{llr}
0 & a_{12}+u a_{11} & a_{13}-u^{2} a_{11} / 2 \\
0 & a_{22}+u a_{21} & a_{23}-u^{2} a_{21} / 2 \\
0 & a_{32}+u a_{31} & a_{33}-u^{2} a_{31} / 2
\end{array}\right)_{J+1}\left(\begin{array}{l}
\delta \rho E \\
\delta \rho u \\
\delta \rho
\end{array}\right)_{J}
\end{aligned}
$$

Thus we related $\delta U_{3+1}$ with $\delta U_{j}$ and the coefficient matrix of $\delta U_{j}$ is modified by absorbing the matrix in Eq. (33).

\section{(e) solid surface:}

We require the normal component of velocity vanishes, 1.e.,

$$
v_{n}=0
$$

and extrapolate the remaining variables from interior.

\section{Solution Procedure}

With the boundary conditions implemented, the first element of the first row and last element of the last row in the block tridlagonal matrix ( $T$ and $M$ ) are essentially eliminated. Since upwind scheme provides diagonal dominance, and assuming $T(M)$ nonsingular, we can get solution for each iteration.

Whether this iteration sequence will converge to the true solution $\left(U^{*}\right)$ depends on wellconditionedness of the matrix $T(M)$ at each iteration, 1.e., if $T(M)$ is 111 -conditioned, the iteration may diverge quickly and will not have a chance to get into the domain $\Omega_{2}$ given in the Theorem shown previously. How fast is the overall convergence rate will then be problem-dependent; problem with mixed signs of eigenvalues in general will need larger number of iterations than that having only one sign. Furthermore, for a given set of initial guess $\left(U^{0}\right)$ and imposed boundary conditions, it is not possible to estimate a priori the domain $\Omega_{2}$ in a nonlinear problem. Therefore in practice the entire iteration sequence may consist of two stages. The initial stage starts out an initial guess, which frequently is assumed using some physical judgment, and moves slowly to $\Omega_{2}$ by some sultable relaxation strategies. We note that one need not use Newton's method in this initial stage and in fact some 1 inearly convergent methods may be preferred and a better implicit operator perhaps is more important. Nevertheless since Newton's method (Eq. (3)) combined with relaxation procedure (Eq. (14)) is already losing feature of quadratic convergence, hence conceptually it may be thought as some lowerorder method. Yet this has the simplicity of using only one solution procedure and quadratic convergence (or nearly) is gotten naturally once $\Omega_{2}$ is reached.

For one-dimensional problem, we write the block tridiagonal matrix $T$ in terms of lower and upper block bldiagonal matrices $L$ and $U$, i.e., LU factorization.

$$
T=L U
$$

A description of the procedure is given in Ref. 13. This is the algorithm that we used to solve onedimensional test problems and may be an optimal one considering number of the algebraic operations and convergence rate of the overall iteration sequence. However for two-dimensional problems, it is not all that clear which solution scheme for a large matrix system, e.g., direct inversion versus iteration, is more suitable insofar as efficiency is concerned. We turn next to outline some possible strategies for the system Eq. (26).

\section{(a) Complete LU Factorization}

Exactly as above in Eq. (35) we write

$$
M=L U
$$

where $L$ and $U$ are of the form

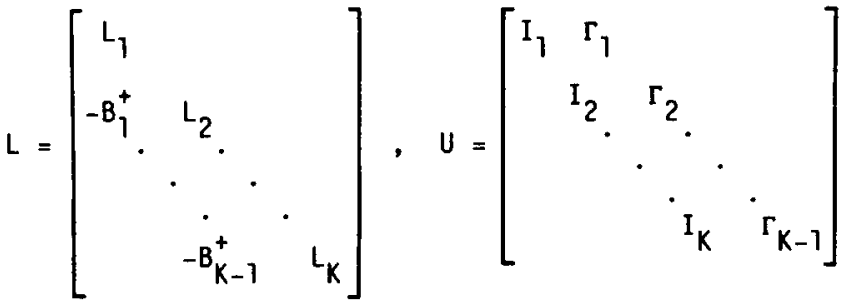

The recursive formula for $L_{k}$ and $\Gamma_{k}$ are given in Ref. 13; inversion and multiplication of block matrices are required along each constant-y line. The direct inversion procedure may be too costly to perform. In what follows we shall discuss some iterative strategles which can be viewed as $\mathrm{m}(>1)$ subiterations for solving each Newton's iteration step. Obviously one need not obtain converged solution at each Newton's iteration which after all has not yet produced "true" solution. But the 
optimal number for $m$ is not known a priori and can be function of many factors, e.g., physical problem considered, grid size, etc. In the application of the present method, we chose $m=1$ for the two-dimenstonal shock reflection problem.

\section{(b) Line Relaxation}

If the off-diagonal blocks in Eq. (27) are taken to the RHS of Eq. (26a), then we are left with a line-decoupled system, 1.e., each constant-y line is in effect independent of each other in so far as solving Eq. (26a) is concerned. Exactly as Eq. (23) in form for each $k(=1,2, \ldots . . . k)$ iline, we now solve one-dimensional block system, hence appropriately called line-relaxation. Since each line is treated as independent, there is no sequence of sweeping (unlike LU factorization) and one can sweep in the same direction for each iteration. However there may be advantages alternating up and down sweeps in successive subfterations, thereby mimicing the complete LU factorization. Furthermore one may skip odd-numbered lines in one sweep and fill in the even-numbered lines in the next sweep, 1.e., so called the zebra scheme. Another possibility is the Gauss-Seidel updating whenever lastest solutions become avallable, hence yielding faster propagation of information. This ine Gauss-Seidel relaxation was used in the present paper.

\section{(c) Point Relaxation}

To further reduce matrix operations, but at the expense of slower propagation of information, all off-diagonal blocks in $M$ and $T_{k}$ are put on RHS. An essentially point-decoupled, termed point relaxation, system is resulted. Similar in spirit to the zebra scheme, a checkerboard scheme can be employed in which points are solved by skipping immediate neighboring points in one sweep and filling in those just skipped in the next sweep. Again one can also combine Gauss-Seidel updating.

\section{Results}

Computational tests of the present method were made for one- and two-dimensional flows. Four cases of one-dimensional nozzle flows were calculated with different inflow and outflow conditions. A calculation of oblique shock reflection was also made. In all calculations presented here untform grids were used. Inflow conditions were also assigned to be the initial guess for the iterative process. The error is taken as the sum of relative absolute changes of all variables at all grid points, i.e.,

error $=\sum_{g r i d s} \sum_{i}^{p} \frac{\left|\delta U_{i}\right|}{\left|U_{i}\right|} /$ (no. of grid points)

so that the convergence of the entire discretized system, rather than a single component (variable) is measured.

In each case calculated we shall present the results which were obtained by using combinations of (1) first- and second-order differencings and (2) Steger-Warming and Van Leer splittings on the
RHS explicit operator. Their effects on accuracy and convergence will be discussed accordingly.

(a) One-dimensional nozzle flows

Two geometries were considered, the area distributions are given by:

Divergent nozzle,

area $=1.398+0.347 \tanh (0.8 x-4), 0<x<10$

Convergent-divergent nozzle,

$$
\text { area }= \begin{cases}1.75-0.75 \cos (x-5) \pi / 5 & 0 \leq x \leq 5 \\ 1.25-0.25 \cos (x-5) \pi / 5 & 5 \leq x \leq 10\end{cases}
$$

We note that since the curvature of the convergentdivergent nozzle is discontinuous at the throat. the flow variables $(p, \rho, u)$ have discontinutties in the first derivative. The grid size $(\Delta x)$ was 0.1 .

Figures 1 show the calculated pressure distributions for fully supersonic flow in the divergent nozzle, together with exact solution denoted by the solid line. Both first-and second-order methods gave virtually the same results. It is evident that a truly quadratic convergence was obtained using the first-order differencing (error was reduced by $10^{-14}$ in seven iterations); here $\omega$ is the relaxation factor appearing in Eq. (15). However the second-order differencing appeared to achleve quadratic convergence initially, but changed to much slower rate. This peculiar behavfor was not understood and is currently under study.

We now present the case with a shock, i.e., the outflow is subsonic, in Fig. 2. The first order upwinding produced monotonic behavior; the Steger-Warming splitting smeared the shock more than the Van Leer splitting did, but was about twice as fast in convergence rate. The difference in convergence rate could be attributed to the fact that the implicit operator remained unchanged (1.e.. Steger-Warming splitting) the explicit operators used either splitting. The $\omega$ was set equal to 0.5 initialiy and increased according to the equations:

$$
\omega=\omega+0.1 \mathrm{n} / \mathrm{n}_{0} \text { if } \operatorname{MOD}\left(n, n_{0}\right)=0
$$

and

$$
\omega=\min (\omega, 1.0)
$$

where $n_{0}$ is the interval of iterations in which $\omega$ is kept fixed. The formula was arbitrarily chosen; better convergence can be gotten when the relaxation process is better understood. The second-order differencing produced much sharper shock, but with unwanted oscillations at both upstream and downstream of the shock. The convergence rate was about half of the first-order method. 
Next we looked at flows in the convergentdivergent nozzle. Figures 3 show the fully subsonic case. Both splitting gave virtualiy identical results except differences in convergence; second-order differencing was clearly superior in accuracy and also nearly equal in convergence rate, contrary to the case in Fig. 1 .

Figures 4 display the case involving a shock; the first-order method clearly lacked spatial resolution. The Van Leer splitting gave much better results in the supersonic region but overpredicted in the inflow subsonic region. The second-order method obviously showed much improved accuracy, but at the expense of slower convergence rate. The dispersive error near the shock was observed in both splittings; the discontinuity at the sonic point in the Steger-Warming splitting was evident and did not appear in the Van Leer splitting, which was designed to yield smooth transition of splitting at sonic point.

Finally we turn to the problem of regular reflection of a two-dimenstonal oblique shock wave with free stream Mach number 2.9 and shock angle $29^{\circ}$. The computation domain was divided uniformly with 61 by 21 grids over $0.0<x<4.0$, and $0.0<y<1.0$, the Steger-Warming flux splitting was used for calculating this case. Figure 5 gives the pressure distributions at the wall, using the first-order upwinding. The shocks evidently were smeared to a large extent. While the static pressure showed monotonic property, the total pressure (or a measure of entropy) displayed overshoot followed by a recovery. The convergence history showed a drastic reduction in error after an initial stage. Figure 6 shows the results obtained by the use of second-order upwinding (boundary conditions remained first-order approximation). Here we see an overexpansion in pressure just ahead of shock foot, followed by a smooth increase. The spatial resolution was clearly much improved over the first-order method. The overexpansion was also accompanied by the increase of total pressure, not seen in the first-order method. The convergence history again showed two distinct stages, confirming the theorem and speculation given in section 1 . The convergence history for the error to reduce $10-10$ is roughly the same for both first-and second-order differencings. The Mach contours are depicted in Fig. 6(d).

\section{Concluding Remarks}

We summarize that an efficient method has been proposed to find steady solution by solving the steady Euler equations. The iterative scheme necessary for solving a coupled nonlinear system was based on Newton's method and an updating (relaxation) procedure. A theorem was shown that the Newton method applied to the system of differential equations gave a quadratic convergence provided the approximation to the true solution is sufficiently close. The first and second deriva$t$ ives of flux vectors were shown to define the domain of the quadratic convergence $\left(\Omega_{2}\right)$. Upwind differencing was necessary to construct the implicit operator for providing the iteration sequence. We employed the true Jacobians resulting from the Steger-Warming spitting in approximating the implicit operator. Both Steger-Warming's and Van Leer's splittings however were used on the explicit operator, combined with the first-and second-order differencings. One-dimensional nozzle flows and two-dimensional shock reflection problem were calculated, specific emphases were placed on the convergence rate and accuracy.

We are continuing to make in-depth investigation of the present method, specially as to the increase of efficiency and application to more complex problems. A high resolution scheme has been implemented 14 and the results will be presented elsewhere.

\section{Acknowledgment}

The author wishes to thank Dr. R. Chima for the help in using graphic program and Dr. H. Huynh for stimulating discussions during preparation of the manuscript.

\section{Appendix A}

We show the true Jacoblans which are derived from the split flux vectors given by Steger and Warming 11 in what follows.

As defined previously

$$
\begin{aligned}
F=F^{+}+F^{-}, & G=G^{+}+G^{-} \\
A \equiv \partial F / \partial U, & B \equiv \partial G / \partial U \\
A^{ \pm} \equiv \partial F^{ \pm} / \partial U, & B^{ \pm}=\partial G^{ \pm} / \partial U \\
|F|=F^{+}-F^{-}, & |G|=G^{+}-G^{-}
\end{aligned}
$$

Hence $|F|=F$ for $u>c$ and $|F|=-F$ for $u<-c$.

Let

$$
|A| \equiv A^{+}-A^{-}
$$

hence

$$
A^{ \pm}=(A \pm|A|) / 2
$$

From Eq. (AI) we have

$$
|A|=\frac{\partial|F|}{\partial U}=\frac{\partial}{\partial U}\left(F^{+}-F^{-}\right)=\left\{\hat{a}_{i j}\right\}
$$

Since the fluxes $F$ and $G$ are split according to Eqs. (23) and (24), a straightforward substitution and differentlation yields, after tedious algebra, for $|u|<c$ :

$$
\begin{gathered}
\hat{a}_{11}=\alpha E, \quad \alpha \equiv \frac{\gamma-1}{2 c} \\
\hat{a}_{12}= \pm B-\alpha u, \quad B=(\gamma-1) / \gamma \\
\hat{a}_{13}=-\alpha v \\
\hat{a}_{14}=\alpha
\end{gathered}
$$




$$
\begin{aligned}
& \hat{a}_{21}=\mp \beta u^{2}+2 \alpha u\left(-e+q^{2} / 2\right), q^{2}=u^{2}+v^{2} \\
& \hat{a}_{22}=2\{ \pm B u+c / \gamma\}-2 \alpha u^{2} \\
& \hat{a}_{23}=-2 \alpha u v \\
& \hat{a}_{24}=2 \alpha u \\
& \hat{a}_{31}=\mp \beta u v+\alpha v\left(-e+\frac{q^{2}}{2}\right) \\
& \hat{a}_{32}= \pm \beta v-\alpha u v \\
& \hat{a}_{33}= \pm \beta u+c / \gamma-\alpha v^{2} \\
& \hat{a}_{34}=\alpha v \\
& \hat{a}_{41}=E\left[\alpha\left(3 u^{2}+v^{2}\right)+3 c\right] / 2 \\
& -\left\{q^{2}[ \pm B u+c / \gamma]+2 c\left[u^{2}+c^{2} /(\gamma-1)\right]\right\} \\
& \hat{a}_{42}=B\left[ \pm q^{2} / 2+u^{2}\right]+3 u c / \gamma-u\left[\alpha\left(3 u^{2}+v^{2}\right)+3 c\right] / 2 \\
& \hat{a}_{43}=v[ \pm B u+c / \gamma]-v\left[\alpha\left(3 u^{2}+v^{2}\right)+3 c\right] / 2 \\
& \hat{a}_{44}=\alpha\left(3 u^{2}+v^{2}\right) / 2+3 c / 2
\end{aligned}
$$

\section{References}

3. Briley, W.R. and McDonald, H., "Solution of the Multidimensional Compressible Navier-Stokes Equations by a Generalized Implicit Method," Journal of Computational Physics, Vol. 24 , No. 4, Aug. 1977, pp. 372-397.

4. Beam, R.M. and Warming, R.F., "An Implicit Factored Scheme for the Compressible NavierStokes Equations," AIAA Journal, Vol. 16, No. 4, Apr. 1978, pp. 393-402.

5. Jameson, A. and Baker, T.J., "Multigrid Solution of the Euler Equations for Aircraft Configurations," AIAA Paper 84-0093, Jan. 1984.

6. MacCormack, R.W., "Current Status of Numerical Solutions of the Navier-Stokes Equations," AIAA Paper 85-0032, Jan. 1985.

7. Chakravarthy, S.R., "Relaxation Methods for Unfactored Implicit Upwind Schemes," AIAA Paper 84-0165, Jan. 1984.

8. Thomas, J.L. and Walters, R.W. "Upwind Relaxation Algor thms for the Navier-Stokes Equations," AIAA Paper 885-1501, July 1985.

9. Walters, R.W. and Dwoyer, D.L., "An Efficient Iteration Strategy for the Solution of the Euler Equations," AIAA Paper 85-1529, July 1985.

10. Dahlquist, G. and Björck, A., Numerical Methods (translated by $N$. Anderson), Prentice-Hall, 1974.

11. Steger, J.L. and Warming, R.F., "Flux Vector Splitting of the Inviscid Gasdynamic Equations with Application to Finite Difference Methods," Journal of Computational Physics, Vol. 40, No. 2, Apr. 1981, pp. 263-293.

12. Van Leer, B., "Flux-Vector Splitting for the Euler Equations," Elghth International Conference on Numerical Methods in Fluid Dynamics, edited by E. Krause, Springer-Verlag, New York, 1982, pp. 507-512.

13. Isaacson, E. and Keller, H.B., Analys is of Numerical Methods, John Wiley and Sons, New York, 1966.

14. Liou, M.-S., "A Generalized Formulation of High Resolution Schemes," accepted for presentation at the First World Congress on Computational Mechanics, Austin, TX, September 1986. 

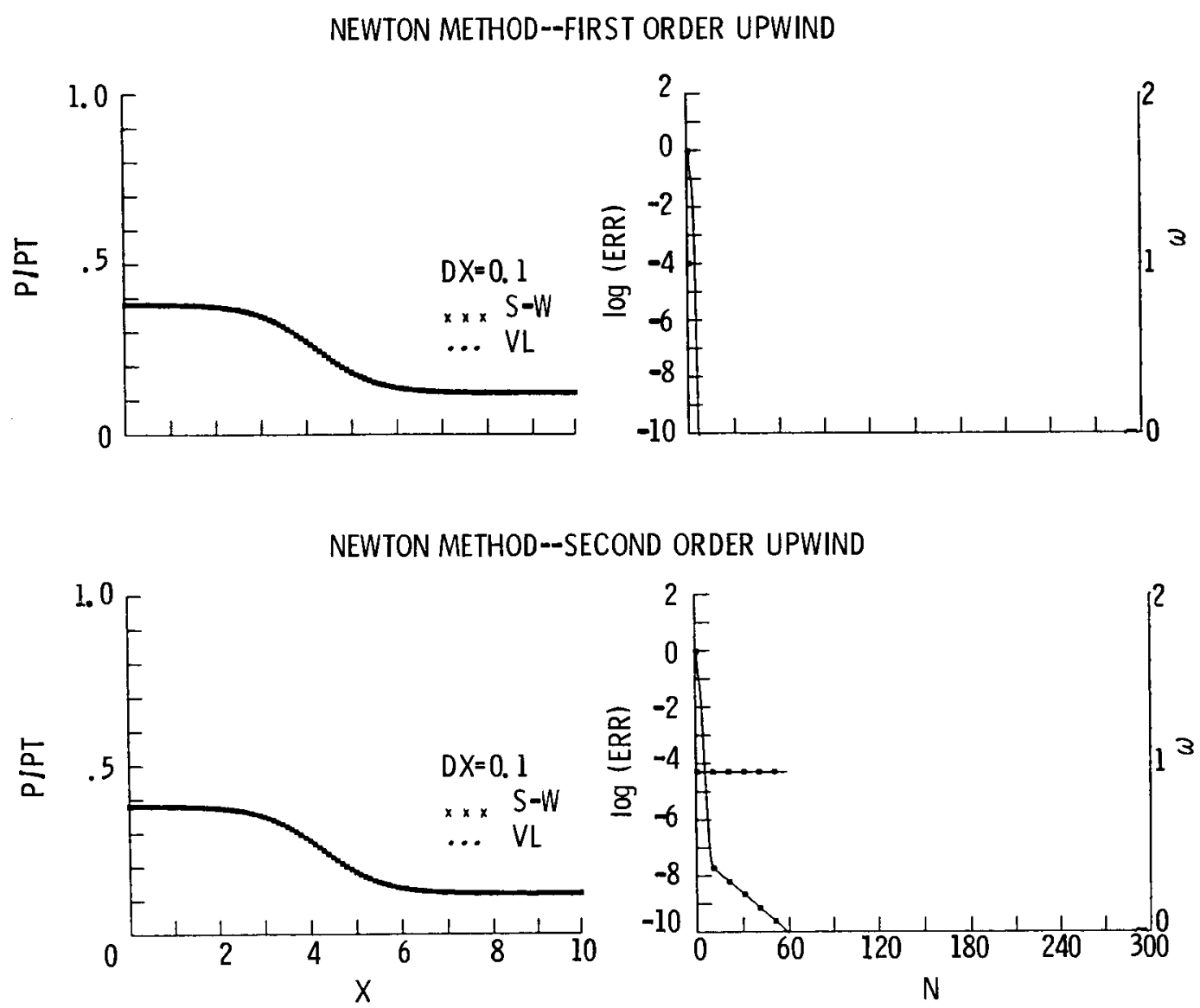

Figure 1. - Static pressure and convergence history for divergent nozzle, $M_{\infty}=1.26$. 

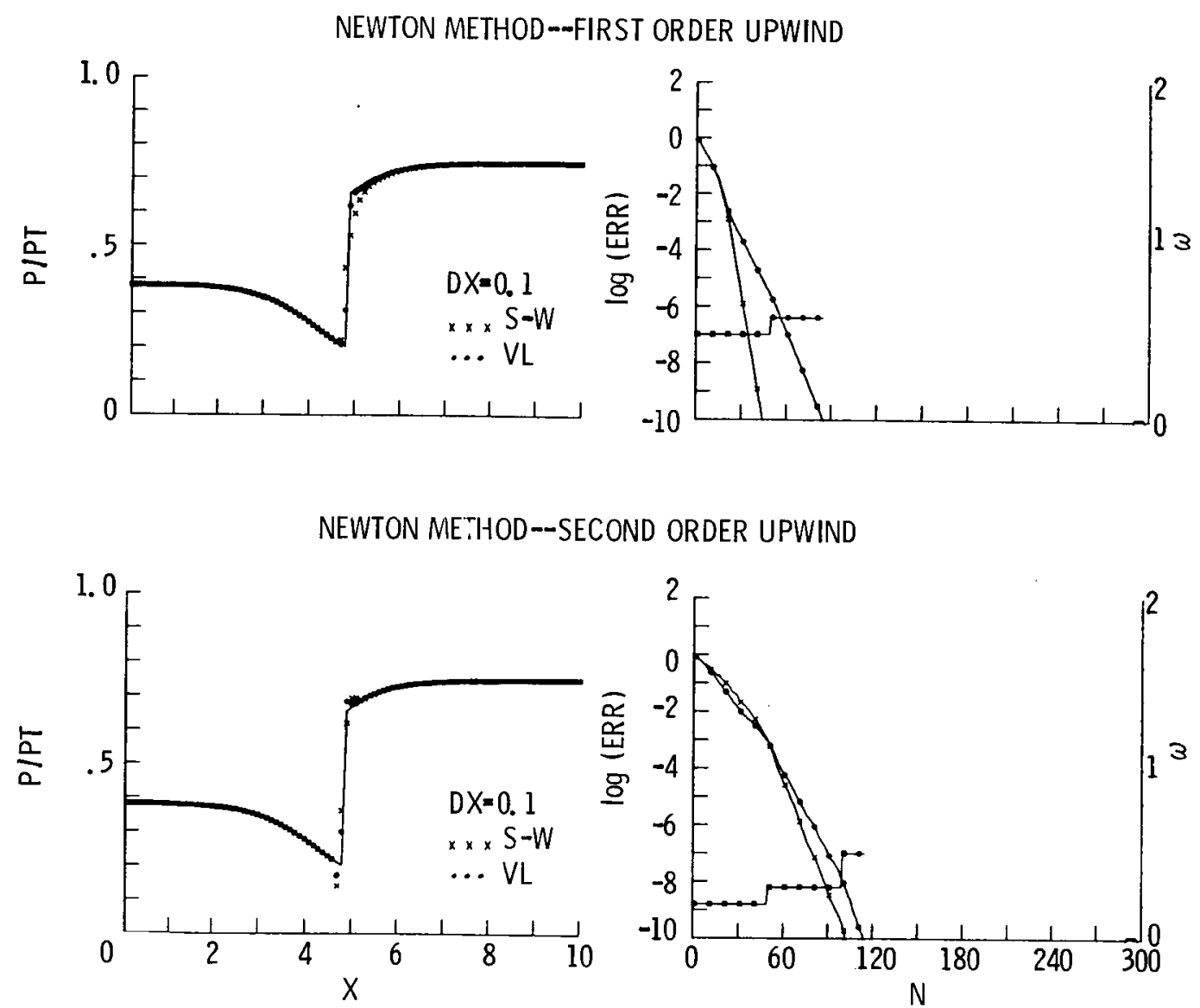

Figure 2. - Static pressure and convergence history for divergent nozzle, $M_{\infty}=1.26$, $\mathrm{P}_{\text {exit }} / \mathrm{P}_{\mathrm{t}}=0.746$. 
NEWTON METHOD--FIRST ORDER UPWIND
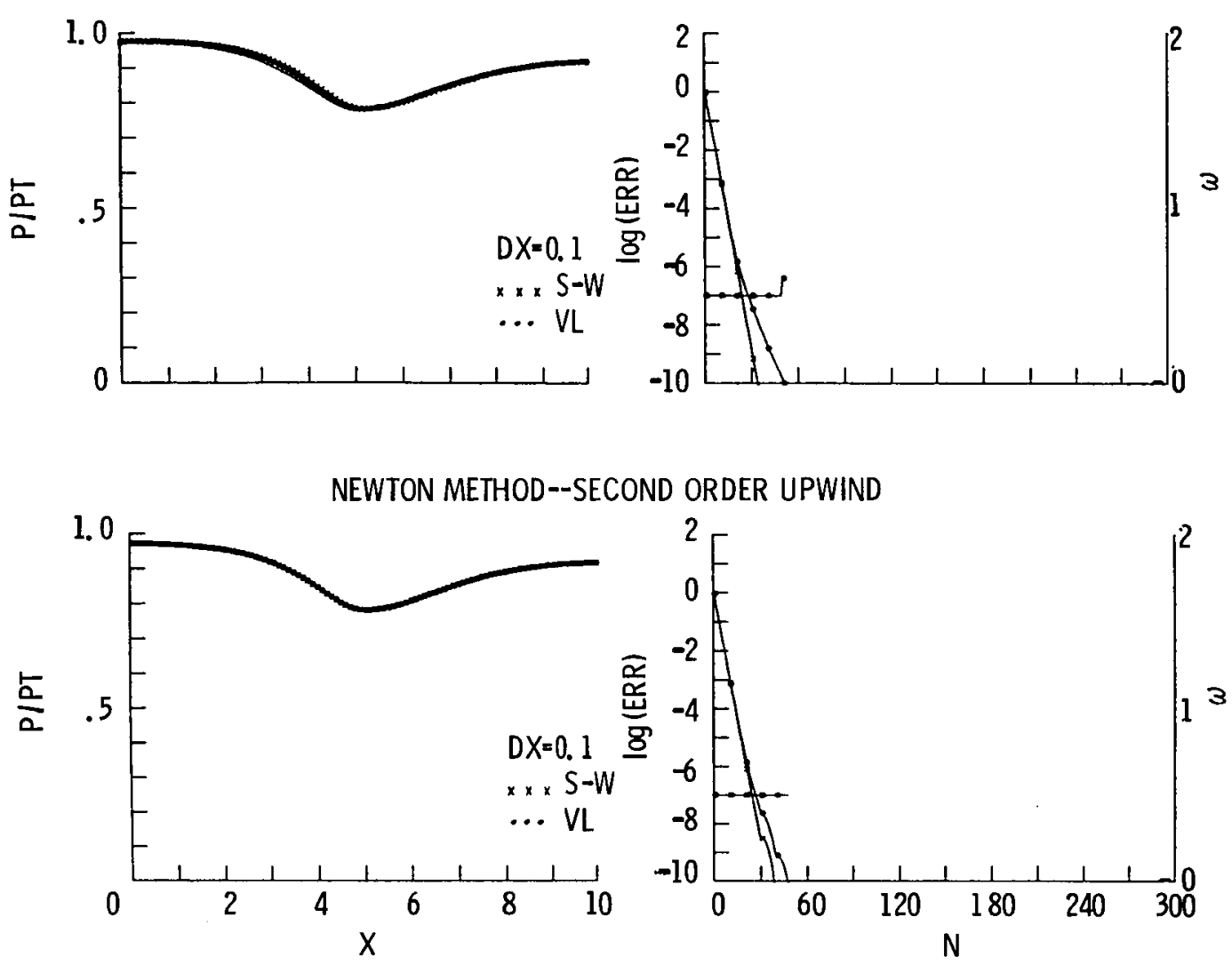

Figure 3. - Static pressure and convergence history for convergent-divergent nozzle, $M_{\infty}=0.2, P_{\text {exit }} / P_{t}=0.9188$. 
NEWTON METHOD--FIRST ORDER UPWIND
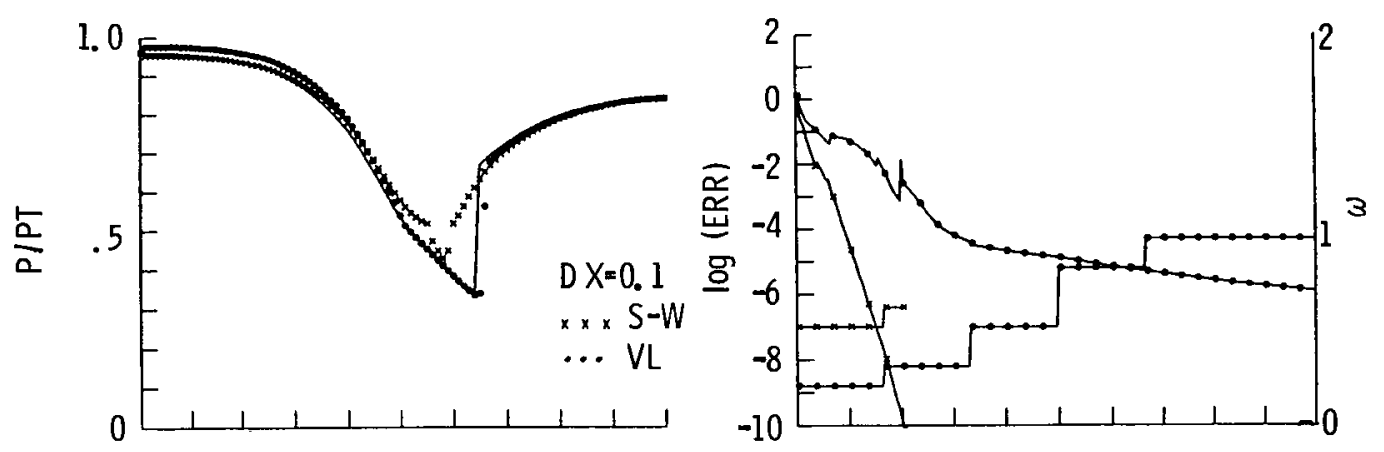

\section{NEWTON METHOD--SECOND ORDER UPWIND}
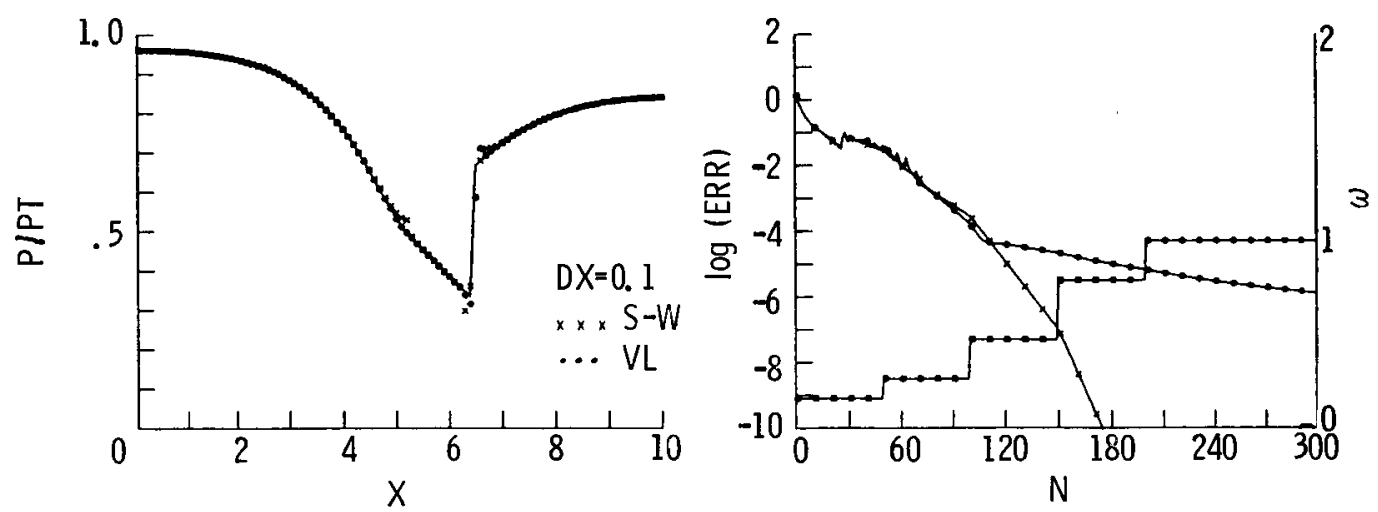

Figure 4. - Static pressure and convergence history for convergent-divergent nozzle, $M_{\infty}=0.2395 ; P_{\text {exit }} / P_{t}=0.84$. 

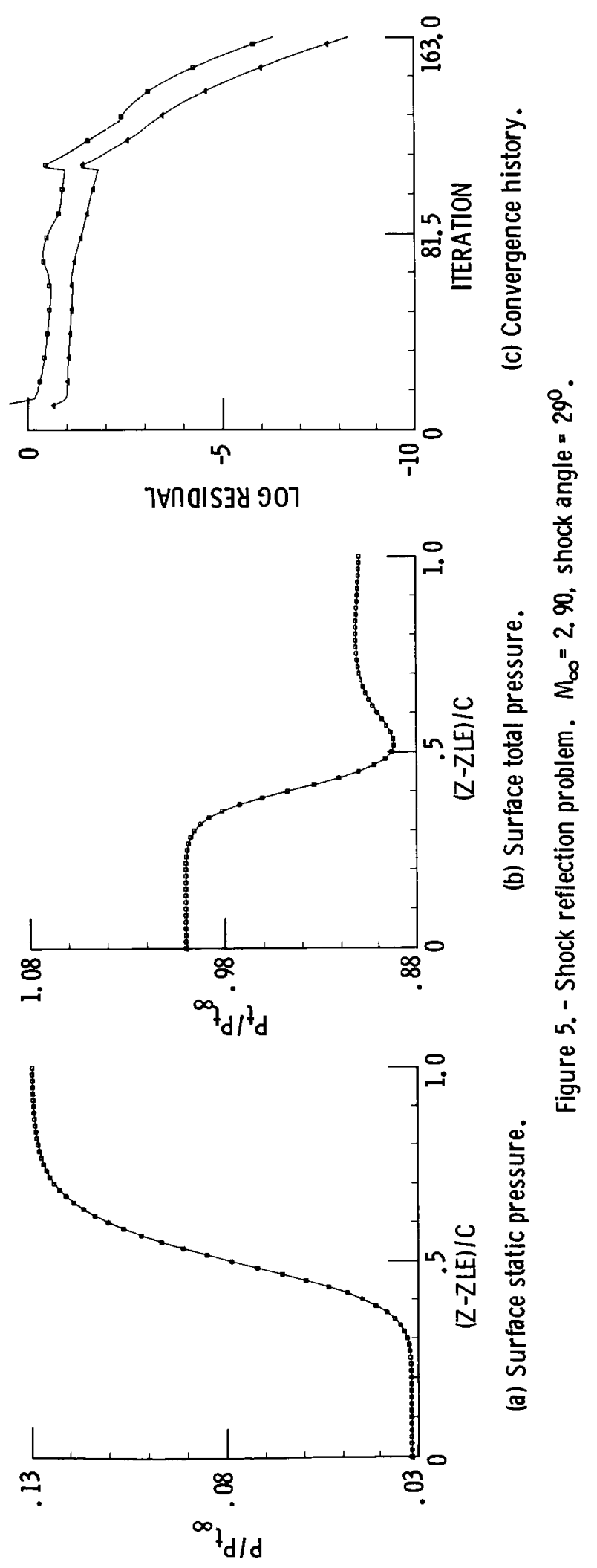

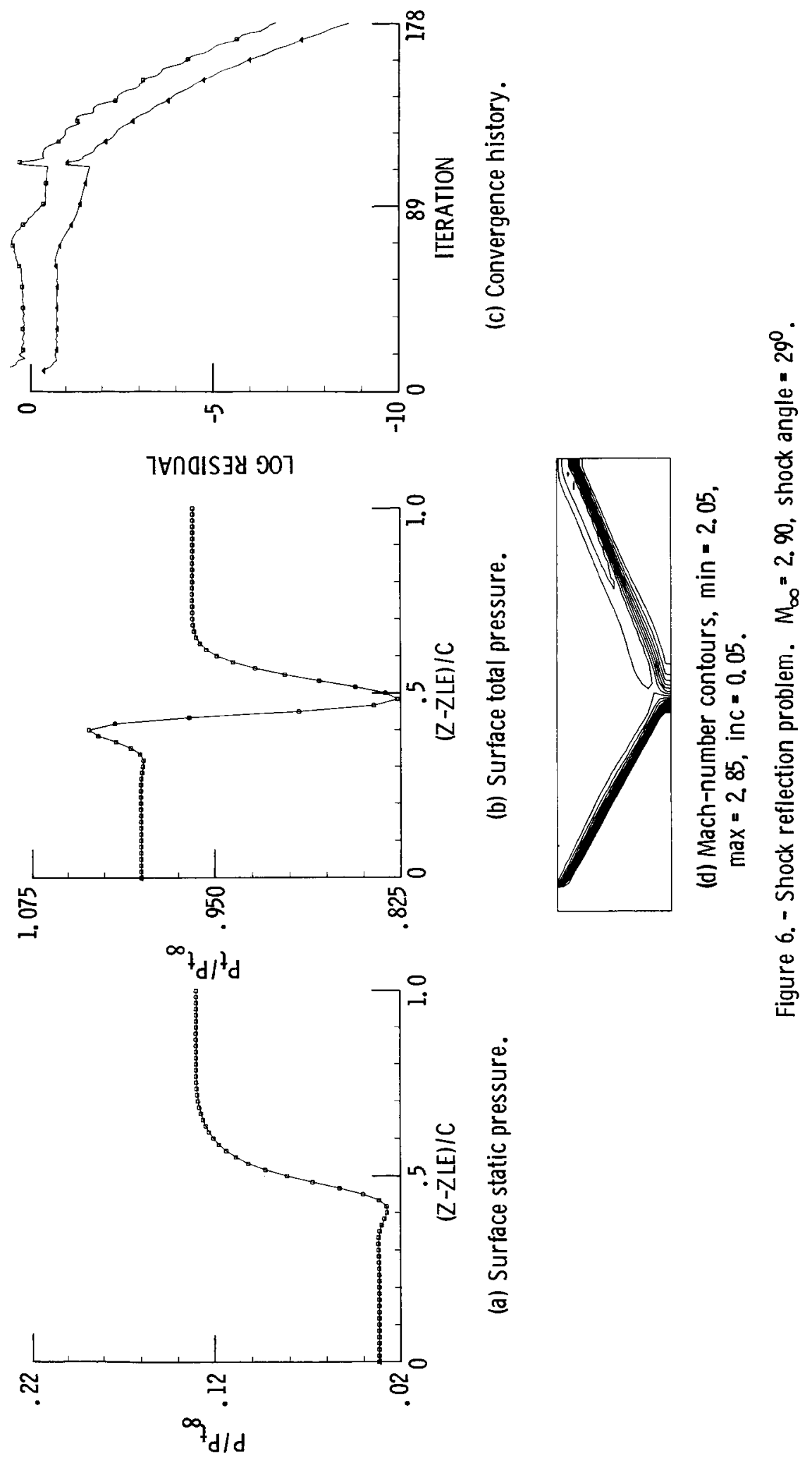


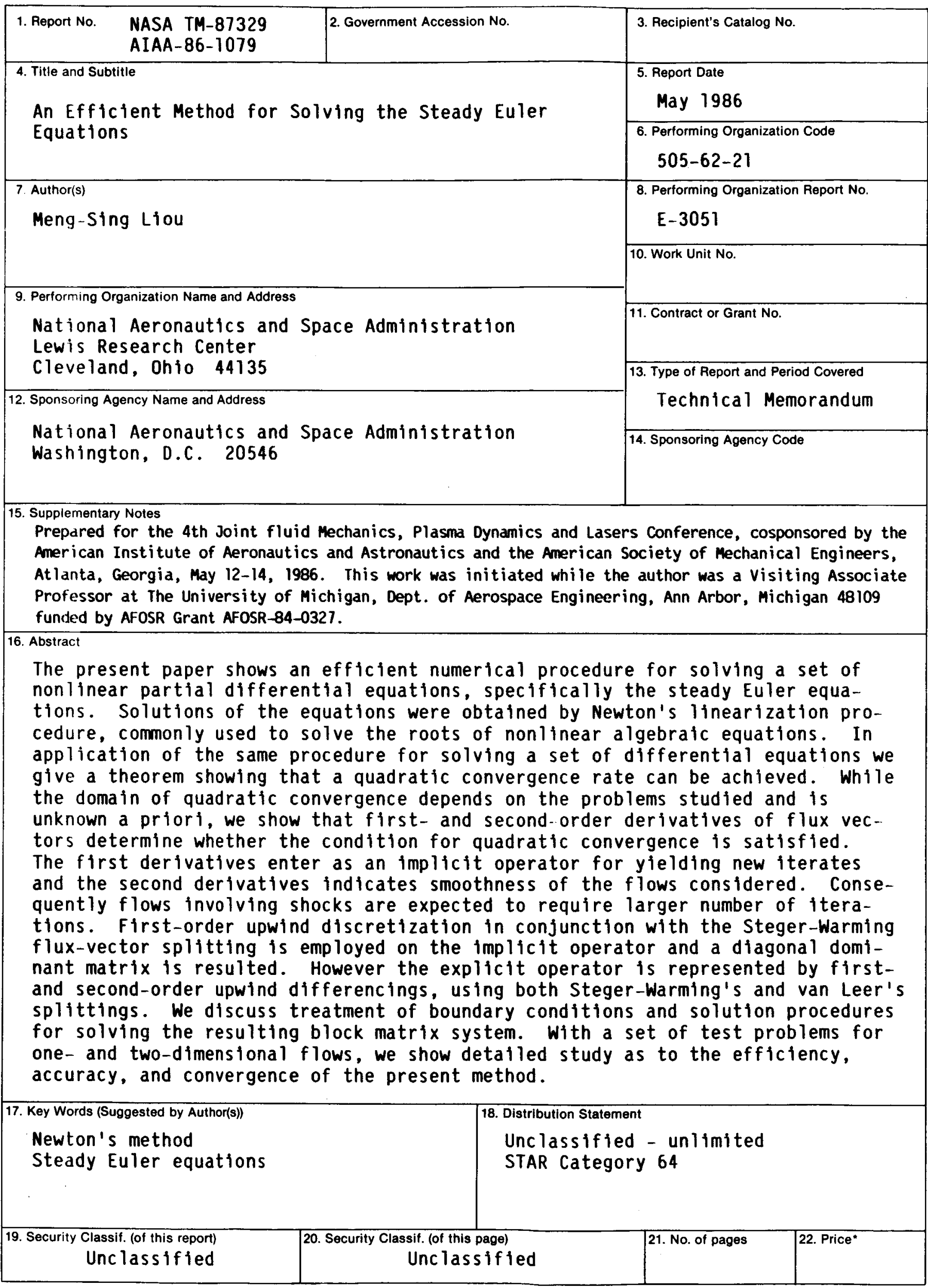

*For sale by the National Technical Information Service, Springfield, Virginia 22161 